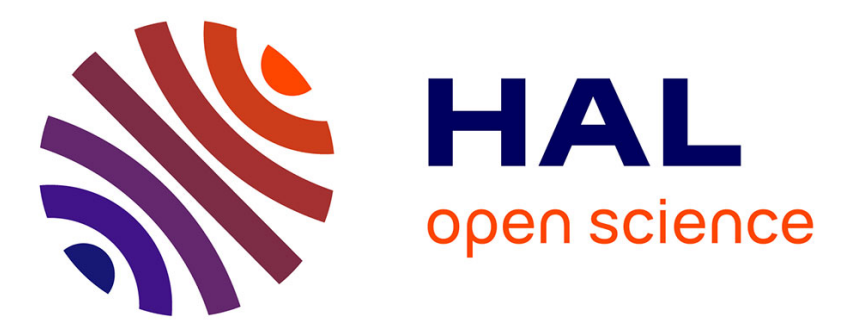

\title{
Magnetic anisotropy and magnetic domain structure in C-doped Mn5Ge3
}

\author{
L.-A. Michez, F. Virot, Matthieu Petit, R. Hayn, L. Notin, Olivier Fruchart, \\ Vasile Heresanu, Matthieu Jamet, Vinh Le Thanh
}

\section{- To cite this version:}

L.-A. Michez, F. Virot, Matthieu Petit, R. Hayn, L. Notin, et al.. Magnetic anisotropy and magnetic domain structure in C-doped Mn5Ge3. Journal of Applied Physics, 2015, 118 (4), pp.043906. 10.1063/1.4927423 . hal-01206141

\section{HAL Id: hal-01206141 https: / hal-amu.archives-ouvertes.fr/hal-01206141}

Submitted on 28 Sep 2015

HAL is a multi-disciplinary open access archive for the deposit and dissemination of scientific research documents, whether they are published or not. The documents may come from teaching and research institutions in France or abroad, or from public or private research centers.
L'archive ouverte pluridisciplinaire HAL, est destinée au dépôt et à la diffusion de documents scientifiques de niveau recherche, publiés ou non, émanant des établissements d'enseignement et de recherche français ou étrangers, des laboratoires publics ou privés. 


\title{
Magnetic anisotropy and magnetic domain structure in C-doped $\mathbf{M n}_{5} \mathbf{G e}_{3}$
}

\author{
L.-A. Michez, ${ }^{1, a)}$ F. Virot, ${ }^{2}$ M. Petit, ${ }^{1}$ R. Hayn, ${ }^{2}$ L. Notin, ${ }^{3,4}$ O. Fruchart, ${ }^{5,}{ }^{6}$ V. Heresanu, ${ }^{1}$ M. Jamet, ${ }^{3,4}$ and V. \\ Le Thanh ${ }^{1}$ \\ 1) Aix-Marseille Université, CNRS, CINaM UMR 7325, 13288 Marseille, France \\ 2) Aix-Marseille Université, CNRS, IM2NP UMR 6242, 13397 Marseille, France \\ ${ }^{3)}$ Univ. Grenoble Alpes, INAC-SP2M, F-38000 Grenoble, France \\ 4) INAC/SP2M, CEA-Grenoble, Grenoble, France \\ 5) Univ. Grenoble Alpes, Inst NEEL, F-38000 Grenoble, France \\ ${ }^{6)}$ CNRS, Inst NEEL, F-38042 Grenoble, France
}

(Dated: 28 September 2015)

Magnetic properties of $\mathrm{Mn}_{5} \mathrm{Ge}_{3} \mathrm{C}_{0.7}$ thin films grown by molecular beam epitaxy have been studied. SQUIDVSM measurements and magnetic force microscopy have been used to probe the magnetic state and determine the relevant magnetic parameters. The results are supported by a combination of improved Saito's and Kittel's models. The moderate perpendicular magnetic anisotropy $\left(Q_{e x p}=\frac{2 K_{u}}{\mu_{0} M_{S a t}^{2}} \approx 0.2\right)$ leads to a stripe domain structure for film thicknesses above $28 \mathrm{~nm}$. For thinner films, the magnetization lies in-plane. The uniaxial magnetocrystalline constant has been found to be much weaker than in $\mathrm{Mn}_{5} \mathrm{Ge}_{3}$ and is assigned to hybridization effect between the $\mathrm{Mn}$ and $\mathrm{C}$ atoms.

PACS numbers: $75.70 . \mathrm{Kw}, 75.50 . \mathrm{Cc}, 75.30 . \mathrm{Gw}$

Keywords: magnetic perpendicular anisotropy, stripe domains, $\mathrm{Mn}_{5} \mathrm{Ge}_{3}, \mathrm{MFM}$

\section{INTRODUCTION}

Recently, much attention has been devoted to manganese germanides for their potential applications in spintronics devices. These materials can be directly integrated into the silicon mainstream technologies and represent indeed a new route to develop the beyond complementary metal-oxide-semiconductor (CMOS) technology. Among them, the $\mathrm{Mn}_{5} \mathrm{Ge}_{3}$ compound is particularly promising due to its high crystalline quality and its ability to be epitaxially grown on Ge(111). Theoretically, this heterostructure should permit direct injection of the spin-polarized current by tunnelling from the $\mathrm{Mn}_{5} \mathrm{Ge}_{3}$ ferromagnet into the type IV semiconductor. Theoretical ${ }^{1,2}$ and experimental ${ }^{3,4}$ investigations of its electronic structure have evidenced the presence of spin polarization and a low contribution of surface states around the Fermi level. ${ }^{5}$ Recently spin accumulation signals have been measured in $\mathrm{Mn}_{5} \mathrm{Ge}_{3} / \mathrm{Ge}(111)$ heterostructures. ${ }^{6,7}$

The limited Curie temperature (296K) of $\mathrm{Mn}_{5} \mathrm{Ge}_{3}$ greatly hinders the use of this material for potential applications. But it has been demonstrated that the presence of carbon into the $\mathrm{Mn}_{5} \mathrm{Ge}_{3} \mathrm{C}_{x}$ lattice permits to maintain the ferromagnetic order up to nearly $450 \mathrm{~K}$ for $x=0.6-0.7 .^{8}$ Theoretical calculations attribute this behavior to an enhancement of the Mn-Mn interactions mediated by C. ${ }^{9}$ As a further benefit, C also improves the thermal structural stability of $\mathrm{Mn}_{5} \mathrm{Ge}_{3} \cdot{ }^{10}$ However, a complete study of the magnetic properties of the $\mathrm{C}$-doped $\mathrm{Mn}_{5} \mathrm{Ge}_{3}$ is still lacking. The main purpose of this paper is to investigate both the magnetic

a) Electronic mail: michez@cinam.univ-mrs.fr anisotropy and the domain structure of $\mathrm{Mn}_{5} \mathrm{Ge}_{3} \mathrm{C}_{0.7}$ thin films. The sample composition corresponding to $x=0.7$ has been chosen as it corresponds to the $\mathrm{C}$ concentration maximizing the Curie temperature $\left(T_{C}\right)$. The thickness dependence of the magnetic properties has been proved to be very helpful to characterize the magnetic behavior of thin films with perpendicular anisotropy. ${ }^{11,12}$ In this work, we use magnetometry and magnetic force microscopy (MFM) to investigate the magnetic properties of $\mathrm{Mn}_{5} \mathrm{Ge}_{3} \mathrm{C}_{0.7}$ thin films as a function of thickness. Contrary to $\mathrm{Mn}_{5} \mathrm{Ge}_{3}, \mathrm{MFM}$ at room-temperature is made possible in $\mathrm{Mn}_{5} \mathrm{Ge}_{3} \mathrm{C}_{0.7}$ by the Curie temperature being much above room temperature. A reorientation of the magnetization in thin $\mathrm{Mn}_{5} \mathrm{Ge}_{3} \mathrm{C}_{0.7}$ films from in-plane to partly out-of-plane has been shown. Above a critical thickness that has been estimated to $28 \mathrm{~nm}$, a stripe domain structure with perpendicular magnetization has been imaged by MFM. The thickness dependence of the stripe period has been fitted with a combination of improved Saito's and Kittel's models, which allows to determine the material constants. The magnetocrystalline anisotropy in C-doped samples is considerably reduced in comparison with $\mathrm{Mn}_{5} \mathrm{Ge}_{3}$.

\section{EXPERIMENTAL ASPECTS}

A series of $\mathrm{Mn}_{5} \mathrm{Ge}_{3} \mathrm{C}_{0.7}$ thin films were grown in a molecular beam epitaxy system having a base pressure of around $3 \times 10^{-10}$ Torr. Ge(111) substrates were chemically and thermally cleaned prior to the deposition of a $50 \mathrm{~nm}$-thick buffer layer. The solid phase epitaxy (SPE) method was then carried out to fabricate the alloy. This technique consists in co-depositing $\mathrm{C}$ and $\mathrm{Mn}$ on a $\mathrm{Ge}(111)$ substrate at room temperature and in sub- 
sequently heating at around $450^{\circ} \mathrm{C}$. This annealing process aims at activating interdiffusion and phase nucleation and is stopped when the characteristic surface reconstruction of the $\mathrm{Mn}_{5} \mathrm{Ge}_{3}$ phase is detected by in situ RHEED (reflection high-energy electron diffraction) observations. $\mathrm{Mn}$ and $\mathrm{C}$ deposition rates must be cautiously calibrated to respect the $\mathrm{Mn}_{5} \mathrm{Ge}_{3} \mathrm{C}_{0.7}$ stoichiometry. Fluxes of Ge and $\mathrm{Mn}$ that were evaporated using standard effusion cells were measured by a quartz crystal microbalance. Carbon evaporation was carried out using a sublimation source of high-purity pyrolytic graphite; the $\mathrm{C}$ concentration was estimated according to the change of $\mathrm{Si}(001)$ surface reconstructions from $(2 \times 1)$ to $c(4 \times 4)$ upon adsorption of a carbon submonolayer. ${ }^{13}$ The estimated error is $10 \%$. The SPE technique has been largely used to obtain high-quality crystalline $\mathrm{Mn}_{5} \mathrm{Ge}_{3}$ thin films and detailed descriptions of samples preparation can be found in ref. 14 and references therein.

Structural properties of post-grown samples have been investigated using transmission electron microscopy (TEM). In addition, X-ray diffraction (XRD) and reflectivity (XRR) characterizations were carried out using a Panalytical Xpert PRO diffractometer equipped with a copper target with a non-monochromatic $\mathrm{K}_{\alpha}$. The angular resolution is $\approx 0.01^{\circ}$. Films thicknesses have been confirmed by reflectivity and high resolution crosssectional TEM measurements.

Magnetic properties have been investigated using magnetometry and magnetic force microscopy (MFM). Magnetic hysteresis loops at $15 \mathrm{~K}$ and at room temperature and Curie temperatures were measured using a Quantum Design SQUID-VSM. The domain structure of asgrown samples has been investigated using an NT-MDT NTEGRA Aura instrument. Magnetic images have been made in amplitude modulation and monitoring the phase signal, either with the two-pass lift technique, or onepass with a slow feedback and weak interaction with the surface. The peak-to-peak amplitude of the cantilever was in the range $7.5-20 \mathrm{~nm}$, with custom-deposited low-moments tips based on SSS-PPP cantilevers from Nanosensors. Most images were made under atmospheric pressure, while those close to the onset of the stripe structure have been made under a vacuum of 1 Torr to enhance the sensitivity.

\section{RESULTS AND DISCUSSION}

\section{A. Structural analysis}

X-ray diffraction has been carried out on a series of $\mathrm{Mn}_{5} \mathrm{Ge}_{3} \mathrm{C}_{0.7}$ films with thicknesses ranging between 10 and $55 \mathrm{~nm}$. For the sake of comparison, a $68 \mathrm{~nm}$-thick $\mathrm{Mn}_{5} \mathrm{Ge}_{3}$ layer has been characterized alike. Full $\theta-2 \theta$ spectra (not shown here) have been recorded. Apart from the strong substrate reflections, the diffraction lines can be indexed assuming a hexagonal structure just as in $\mathrm{Mn}_{5} \mathrm{Ge}_{3}$. As suggested in previous work, ${ }^{8,9}$ the struc-

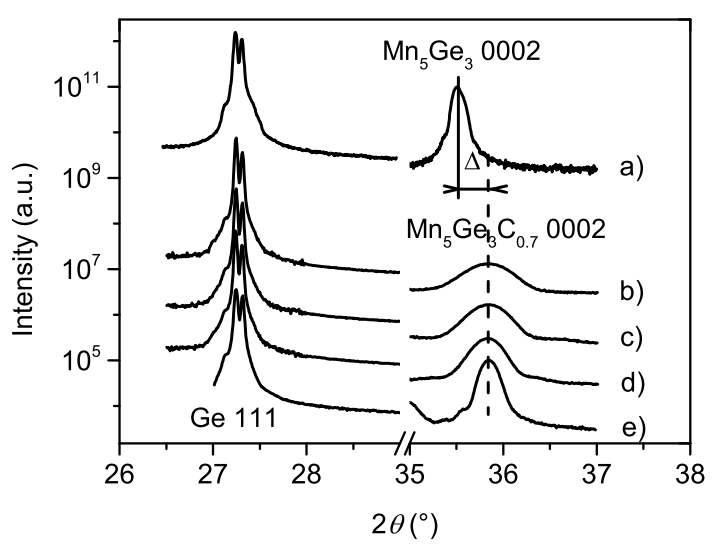

FIG. 1. X-ray diffraction spectra around the Ge 111 and $\mathrm{Mn}_{5} \mathrm{Ge}_{3} 0002$ reflections of a) a $68 \mathrm{~nm}$-thick $\mathrm{Mn}_{5} \mathrm{Ge}_{3}$ film, b) to e) $\mathrm{Mn}_{5} \mathrm{Ge}_{3} \mathrm{C}_{0.7}$ layer with respective thicknesses of $20 \mathrm{~nm}$, $30 \mathrm{~nm}, 40 \mathrm{~nm}$ and $55 \mathrm{~nm}$.

ture of the C-doped films remains unchanged with an hexagonal unit cell containing two sublattices of Mn (denoted $\mathrm{Mn}_{I}$ and $\mathrm{Mn}_{I I}$ ) with different coordination. The $\mathrm{C}$ atoms are likely to occupy the voids at the center of the $\mathrm{Mn}_{\text {II }}$ octahedron. The X-ray diffractogram shows only the $\mathrm{Mn}_{5} \mathrm{Ge}_{3} 0002$ and 0004 reflections. The films are therefore epitaxial and contain no other phases, which means that all the $\mathrm{Mn}$ and $\mathrm{C}$ have been consumed to form a unique phase.

Fig. 1 displays the data around Ge 111 and $\mathrm{Mn}_{5} \mathrm{Ge}_{3}$ 0002 reflections. The peaks are doublets because $K_{\alpha 1}$ and $\mathrm{K}_{\alpha 2}$ can not be dissociated as our XRD system is not monochromatized. The presence of $\mathrm{C}$ has clearly caused a shift of the $\mathrm{Mn}_{5} \mathrm{Ge}_{3} 0002$ reflection towards the higher angles. The lattice parameter along the hexagonal $c$-axis can be determined from the diffraction lines. It corresponds to the $\mathrm{Mn}_{5} \mathrm{Ge}_{3}$ bulk value in the undoped film whereas a $0.9 \%$ lattice compression is observed in the $\mathrm{Mn}_{5} \mathrm{Ge}_{3} \mathrm{C}_{0.7}$ films for all the considered thicknesses. Interestingly, similar lattice variations have been observed in $\mathrm{Mn}_{5} \mathrm{Ge}_{3} \mathrm{C}_{x}$ films grown using different techniques ${ }^{8,15,16}$ and containing different carbon concentrations. ${ }^{16}$ This value accords well to the lattice parameters that have been theoretically calculated in reference 9 in a relaxed $\mathrm{Mn}_{5} \mathrm{Ge}_{3} \mathrm{C}_{x}$ film. In the thickness range considered in this paper, no additional thicknessdependent strain is observed. The diffraction peaks sharpen with increasing the thickness. Their full width at half maximum (FWHM) has been determined by peak fitting taking into account the two wavelengths $K_{\alpha 1}$ and $\mathrm{K}_{\alpha 2}$. The reduced thickness introduces a peak broadening. While the Scherrer formula yields the correct thickness in the $\mathrm{Mn}_{5} \mathrm{Ge}_{3}$ film, the width of the peak in the Cdoped $\mathrm{Mn}_{5} \mathrm{Ge}_{3}$ films lightly underestimates the reduced film thickness. This hints at a slight reduction of crystal coherence coming from the $\mathrm{C}$ introduction. Although TEM images of C-doped samples (not shown here) re- 


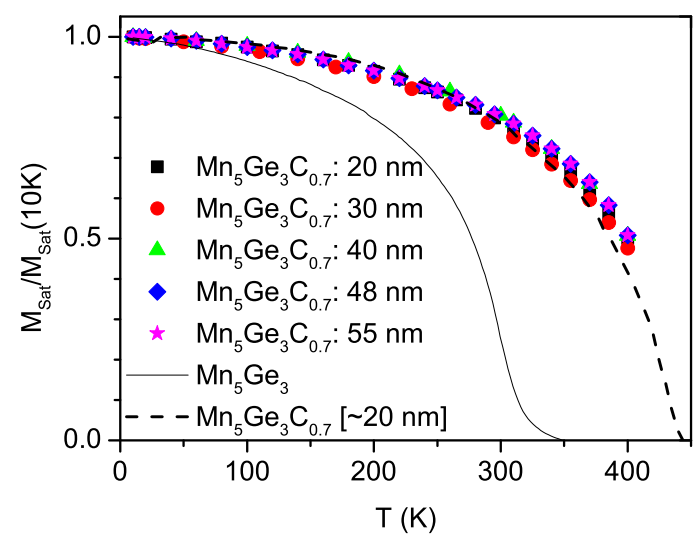

FIG. 2. (color online) Temperature dependence of the saturation magnetization $\left(M_{\text {Sat }}\right)$ recorded in a $1 \mathrm{~T}$-field for a 20 $\mathrm{nm}-$, a $30 \mathrm{~nm}-$, a $40 \mathrm{~nm}-$, a $48 \mathrm{~nm}-$ and a $55 \mathrm{~nm}$-thick film of $\mathrm{Mn}_{5} \mathrm{Ge}_{3} \mathrm{C}_{0.7}$. The grey full line and the black dashed line represent the $M$ - $T$ curves measured on $\approx 20 \mathrm{~nm}$-thin films of respectively $\mathrm{Mn}_{5} \mathrm{Ge}_{3}(\mathrm{x}=0)$ and $\mathrm{Mn}_{5} \mathrm{Ge}_{3} \mathrm{C}_{0.7}(\mathrm{x}=0.7)$ (from reference 14$)$.

veal still a good quality crystal, ${ }^{14,17}$ the presence of $\mathrm{C}$ degrades to some extend the very high crystalline quality by introducing local strain.

\section{B. Magnetic characterizations}

\section{SQUID-VSM magnetometry}

As the magnitude of the Curie temperature $\left(T_{C}\right)$ is directly related to the $\mathrm{C}$ concentration, the temperature dependence of the saturation magnetization $\left(M_{\text {Sat }}\right)$ has been systematically measured in an applied magnetic field of $1 \mathrm{~T}$ and compared to the behavior of the $\mathrm{Mn}_{5} \mathrm{Ge}_{3} \mathrm{C}_{0.7}$ film in reference 14 . The $M$ - $T$ curves displayed in Fig. 2 are overlaid, which demonstrates that all our films contain an equivalent quantity of $\mathrm{C}$ as no finitesize effect on $\mathrm{Tc}$ are expected for such large thicknesses. The $T_{C}$ has been estimated to be $435 \pm 5 \mathrm{~K}$, which is in the uncertainty range of the value found in reference 14 for an equivalent film composition. This composition corresponds to the optimal carbon concentration regarding the magnetic properties.

Fig.3 shows in-plane and out-of-plane hysteresis curves for $20 \mathrm{~nm}-, 30 \mathrm{~nm}$ - and $55 \mathrm{~nm}$-thick $\mathrm{Mn}_{5} \mathrm{Ge}_{3} \mathrm{C}_{0.7}$ films measured at room temperature. The insets of Fig.3 present the in-plane $M-H$ curves measured at $15 \mathrm{~K}$ for $\mathrm{Mn}_{5} \mathrm{Ge}_{3}$ and $\mathrm{Mn}_{5} \mathrm{Ge}_{3} \mathrm{C}_{0.7}$ thin films with similar thicknesses. The first major difference between the magnetic properties of the two alloys concerns the saturation magnetization that is lower in the C-doped films. This peculiarity has been already experimentally observed in Cdoped $\mathrm{Mn}_{5} \mathrm{Ge}_{3}{ }^{8}$ and has been theoretically ascribed to the $\mathrm{C}$ incorporation: hybridization between the $\mathrm{C} 2 p$ -

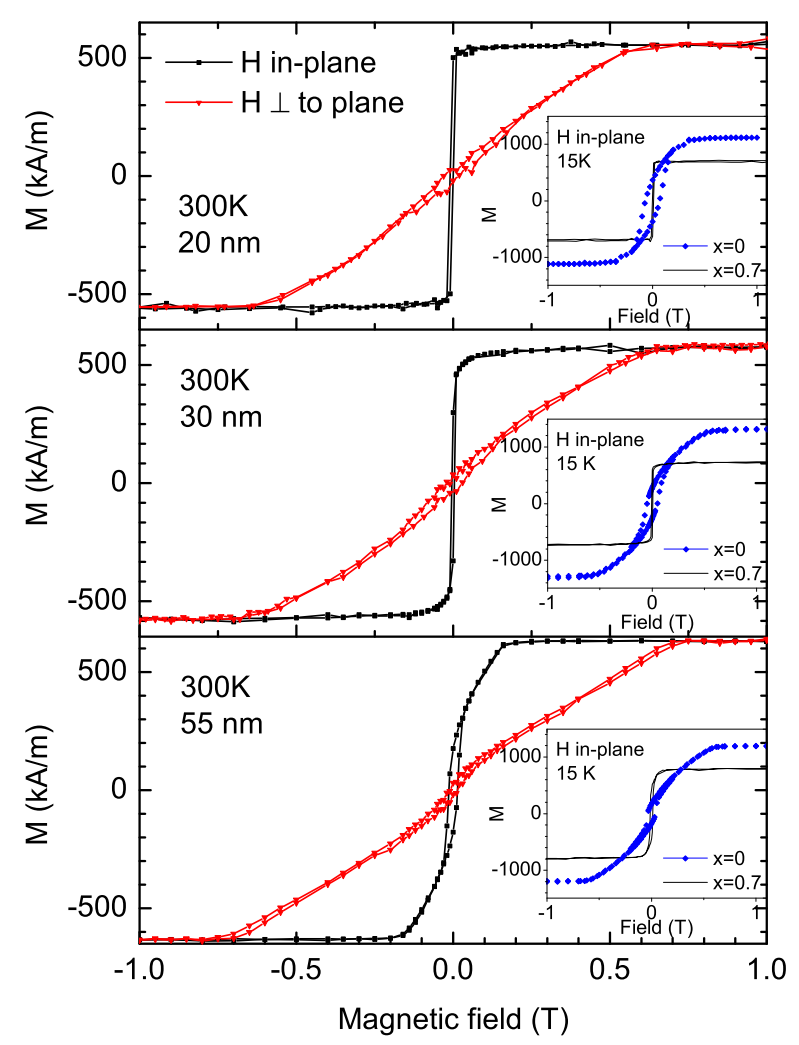

FIG. 3. (color online) Hysteresis loops performed at $300 \mathrm{~K}$ for (a)for $20 \mathrm{~nm}-$, (b) $30 \mathrm{~nm}$ - and (c) $55 \mathrm{~nm}$ - thick samples with the external magnetic field applied in the sample plane (black squares) and perpendicular to it (red triangles). The insets display the in-plane $M-H$ curves performed at $15 \mathrm{~K}$ for $\mathrm{Mn}_{5} \mathrm{Ge}_{3}(x=0)$ and $\mathrm{Mn}_{5} \mathrm{Ge}_{3} \mathrm{C}_{x}(x=0.7)$ thin films of equivalent thickness.

and $\mathrm{Mn}_{I I} 3 d$-states leads to a reduced magnetic moment of $\mathrm{Mn}_{I I} \cdot{ }^{9} \mathrm{In}$ addition, the structure relaxation caused by interstitial atoms decrease the interatomic Mn-Mn distances, slightly reducing the magnetic moments. ${ }^{9}$

The magnetization at saturation has been plotted as a function of thickness in Fig. 4a. The linear behavior of this figure is well described by $M_{\text {Sat }}^{\text {Meas }} \times t_{\text {Film }}=M_{\text {Sat }}^{I n t} \times$ $\left(t_{\text {Film }}-t_{F D L}\right)$, where $M_{\text {Sat }}^{\text {Meas }}$ and $M_{\text {Sat }}^{I n t}$ are respectively the measured and intrinsic magnetizations and $t_{F D L}$ is the thickness of the ferromagnetically dead layer (FDL), which corresponds to the non-zero thickness giving zero magnetization. $M_{\text {Sat }}^{I n t}$ has been estimated to be $(640 \pm 40)$ $\mathrm{kA} \cdot \mathrm{m}^{-1}$ at $300 \mathrm{~K}$ and $(810 \pm 40) \mathrm{kA} \cdot \mathrm{m}^{-1}$ at $15 \mathrm{~K}$.

Similarly to $\mathrm{Mn}_{5} \mathrm{Ge}_{3} / \mathrm{Ge}(111)$ heterostuctures ${ }^{18}$ a FDL has been formed at the interface between the $\mathrm{Ge}(111)$ substrate and the $\mathrm{Mn}_{5} \mathrm{Ge}_{3} \mathrm{C}_{0.7}$ film and its thickness $t_{F D L}$ has been estimated to be $(2.0 \pm 0.5) \mathrm{nm}$ at $300 \mathrm{~K}$ and $(1.3 \pm 0.5) \mathrm{nm}$ at $15 \mathrm{~K}$. This is equal to the value found in $\mathrm{Mn}_{5} \mathrm{Ge}_{3}$ within experimental errors. ${ }^{18} \mathrm{Al}-$ 


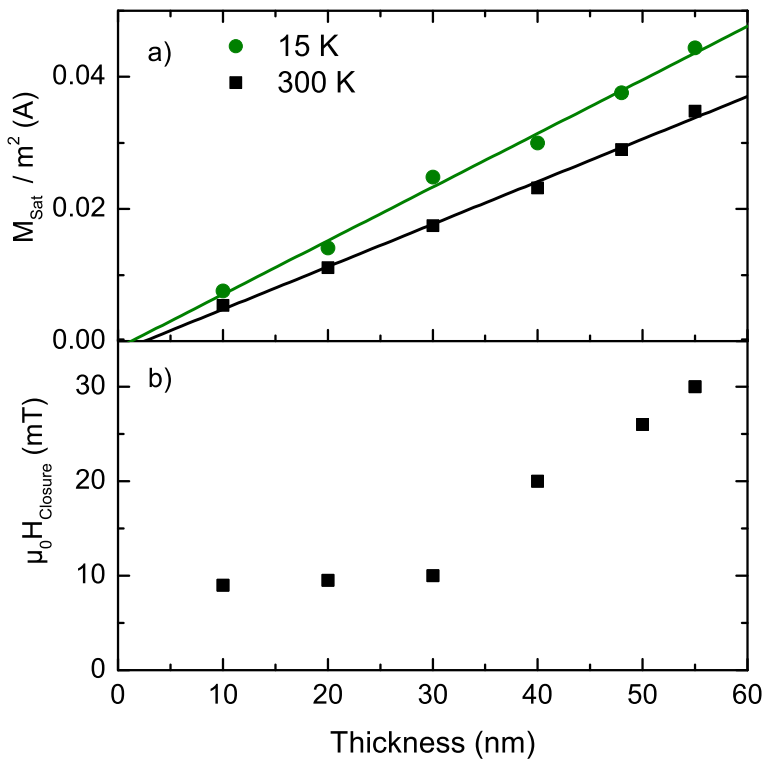

FIG. 4. (color online) a) Saturation magnetic moment per surface area as a function of the film thickness. The slope of the linear fit gives the intrinsic magnetization of $\mathrm{Mn}_{5} \mathrm{Ge}_{3} \mathrm{C}_{0.7}$. b) Thickness dependence of $H_{\text {Closure }}$ corresponding to the field at the end of the opening in the $M-H$ curves.

though no structural gradients are visible on the crosssection high-resolution TEM (HR-TEM) images recorded near the interfaces, ${ }^{14}$ the formation of a non ferromagnetic interfacial alloys is probable. A recent study by scanning tunneling microscopy and x-ray photoelectron spectroscopy indicates an excess of $\mathrm{Ge}$ atoms in the first $\mathrm{Mn} / \mathrm{Ge}$ layer in $\mathrm{Mn}_{5} \mathrm{Ge}_{3}$ films. ${ }^{19}$ Our results suggest that $\mathrm{C}$-doping does not reduce this effect. Recently a very-low-temperature epitaxial growth of $\mathrm{Mn}_{5} \mathrm{Ge}_{3} \mathrm{C}_{0.7}$ has been achieved by reactive deposition epitaxy. ${ }^{16}$ This new technique should considerably limit segregation and diffusion of $\mathrm{Mn}$ at the interface and represent a solution to hamper the FDL formation.

The thickness dependence of the $M-H$ curves in $\mathrm{Mn}_{5} \mathrm{Ge}_{3}$ and $\mathrm{Mn}_{5} \mathrm{Ge}_{3} \mathrm{C}_{0.7}$ thin films follows the same trend. A gradual change is observed in the shape of the in-plane hysteresis loops. ${ }^{18,20}$ The saturation field increases with thickness giving rise to a more canted curve. This effect, particularly visible in the $\mathrm{Mn}_{5} \mathrm{Ge}_{3}$ epilayer, is assigned to a reorientation of the magnetization in thin films with perpendicular anisotropy. For low thicknesses, the magnetostatic anisotropy forces the magnetization in-plane giving rise to a single domain structure. As the thickness increases, the magnetization is expected to progressively develop locally an out-of-plane component, through a so-called weak stripe structure. ${ }^{21}$ However, the critical thickness is different for the two materials. Whereas a stripe domain structure has just been formed in a $20 \mathrm{~nm}-\mathrm{Mn}_{5} \mathrm{Ge}_{3}$ film, ${ }^{18}$ the square inplane $M-H$ curve measured in a $\mathrm{Mn}_{5} \mathrm{Ge}_{3} \mathrm{C}_{0.7}$ layer with an equivalent thickness is characteristic of completely inplane magnetization. As magnetization remains uniform below the critical thickness, the uniaxial anisotropy $K_{u}$ can be determined from the analysis of $M-H$ curves and has been estimated to be $(50 \pm 10) \mathrm{kJ} / \mathrm{m}^{3}$ at $300 \mathrm{~K}$ and $(60 \pm 10) \mathrm{kJ} / \mathrm{m}^{3}$ at $15 \mathrm{~K}$. The values deduced for $30 \mathrm{~nm}-$ thick films are very similar.

In a magnetic film, the strength of the perpendicular magnetic anisotropy (PMA) can be expressed as the ratio between the uniaxial anisotropy and the film shape anisotropy, leading to the definition of the quality factor $Q=\frac{2 K_{u}}{\mu_{0} M_{\text {Sat }}^{2}}$. For t $=20 \mathrm{~nm}, Q_{\text {exp }}$ has been estimated to 0.20 at $300 \mathrm{~K}$ and 0.16 at $15 \mathrm{~K}$. Thin films with moderate $Q$ factor $(Q \leq 1)$ value present specific magnetic configurations with the existence of a critical thickness above which stripe domains are nucleated. In these structures, the magnetization periodically oscillates out-ofplane and domains are separated by Bloch-type domain walls (DWs). Information about such domains can be obtained from the hysteresis curves. For example, $H_{\text {Closure }}$ defined as the magnetic field at the end of the irreversible part of the in-plane hysteresis loop is plotted in Fig.4 as a function of $\mathrm{Mn}_{5} \mathrm{Ge}_{3} \mathrm{C}_{0.7}$ thickness. This value corresponds to the field necessary to align the magnetization within the DWs in the applied field direction. ${ }^{18}$ Little variation in this quantity is noticeable for film thicknesses up to $30 \mathrm{~nm}$, whereas, above this value, $H_{\text {Closure }}$ starts to increase with thickness, along with the onset of canting of the loops. This suggests a modification of the domain structure around $30 \mathrm{~nm}$.

\section{Domain structure studied by MFM}

The zero-field magnetic structure of the as-grown samples before applying any external field has been investigated by magnetic force microscopy. For thicknesses smaller than $30 \mathrm{~nm}$, no magnetic contrast could be observed confirming that the magnetization lies in-plane with domain sizes larger than scan sizes. In contrast, as the film thickness exceeds $30 \mathrm{~nm}$, the images presented in Fig. 5 display a stripe feature, characteristic of alternated regions with at least partly up and down magnetization. The stripe domain appears spontaneously, as a compromise to decrease the magnetocrystalline anisotropy energy by locally tilting magnetization out-of-plane, while closing the flux with alternating up-and-down directions, so as to keep magnetostatic energy moderate. ${ }^{22}$ The magnetic configuration consists of a disordered array of stripes with however well-defined widths, the period of the pattern corresponding to two consecutive domains with magnetization pointing upwards (or downwards). This highly branched stripe configuration has been well described in the literature, ${ }^{23,24}$ and is assigned to defects, thermal meandering of domain walls and magneticfield induced strain. The domain structure pattern may also depend on the magnetic history of the film. ${ }^{12,25}$ The labyrinth structure changes to a parallel-stripe structure 

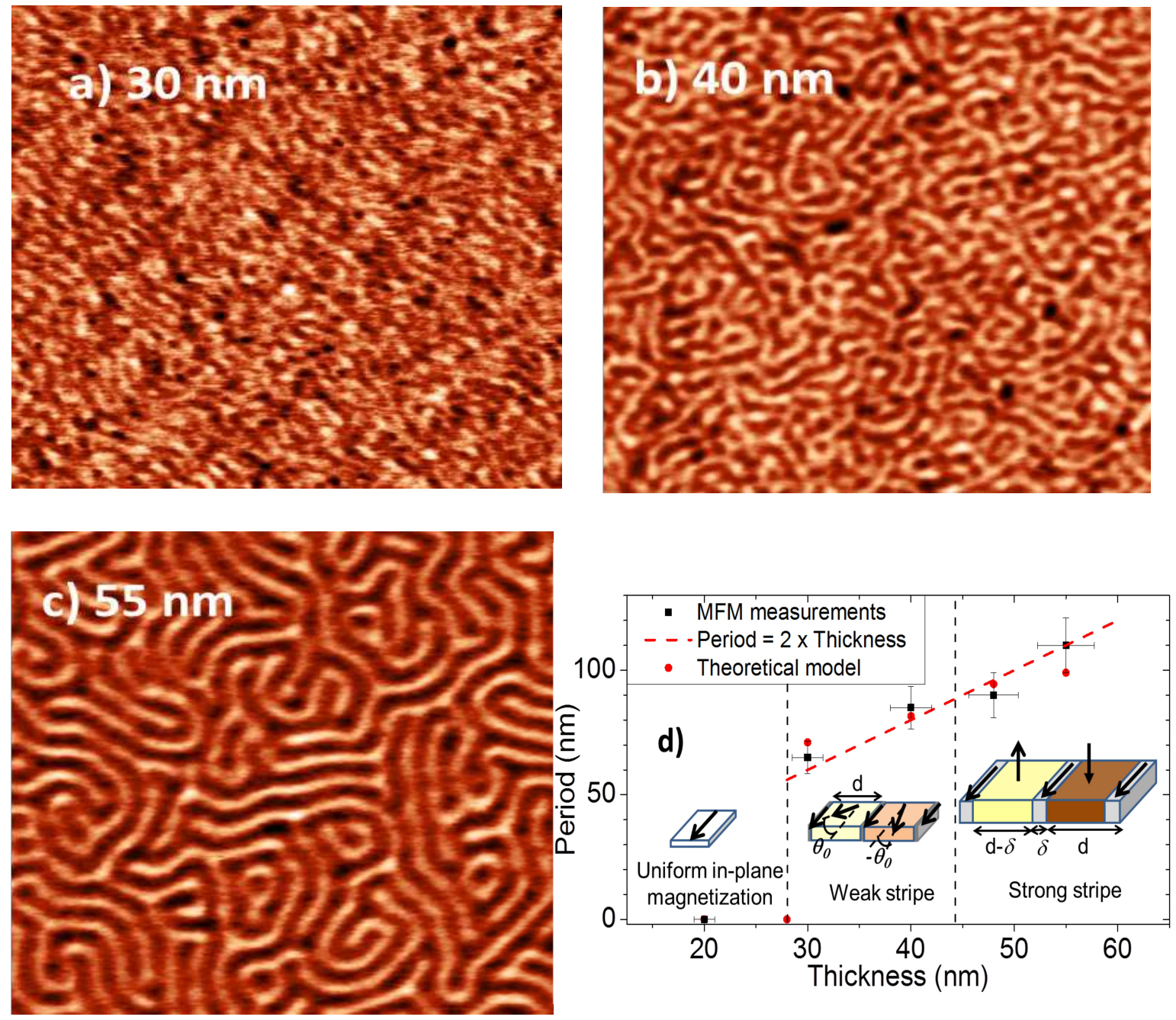

FIG. 5. (color online) Zero applied field MFM images for a) $30 \mathrm{~nm}-$, b) $40 \mathrm{~nm}$ - and c) $55 \mathrm{~nm}$-thick $\mathrm{Mn}_{5} \mathrm{Ge}_{3} \mathrm{C}_{0.7}$ films. All the MFM images have the same size of $2 \mu \mathrm{m} \times 2 \mu \mathrm{m}$ and show the as-grown state. d) Period of magnetic domains as a function of film thickness. Error bars of experimental measurements are set to 10\%. Schematic diagrams of the domain structure deduced from our calculations are represented for each thickness range.

upon in-plane magnetic saturation and coming back to zero field. The domain period remains unchanged during the process of stripe alignment. The average stripe domain period has been determined through an autocorrelation processing and plotted as a function of increasing thickness from 30 to $55 \mathrm{~nm}$ in the bottom panel of Fig. 5 . Both an increase of the period and an enhancement of the magnetic contrast between two consecutive domains with opposite magnetization have been observed. For films with small $Q$ factor $(Q<0.1)$, the period at nucleation is approximately twice the thickness value. ${ }^{26}$ Our data follow relatively well this tendency. In these conditions, the critical thickness below which the magnetization is planar is given by $t_{c r}=2 \pi \sqrt{A / K_{u}}$. Considering a critical thickness of about $30 \mathrm{~nm}$ and the anisotropy constant previously determined, the exchange stiffness in the carbon-doped compound may be estimated to $1.4 \mathrm{pJ} / \mathrm{m}$.
This value is greater than the one found in $\mathrm{Mn}_{5} \mathrm{Ge}_{3}$ (1 $\mathrm{pJ} / \mathrm{m}),{ }^{18}$ which is consistent with the Curie temperature enhancement.

\section{Theoretical model}

A comparison of the magnetic domains characteristics measured by MFM with domain theories is necessary to obtain a good understanding of the domain structure and extract the variation of material parameters with film thickness. For example, in the case of uniaxial thin films, the thickness dependence of the domains period can be well described by a Kittel model, refined to take into account domain walls, which is found to be important for films with thickness comparable to the domain wall width as is the case here. ${ }^{11,27}$ Following Kittel's ap- 
proach, we consider that the magnetization within domains is uniformly out-of-plane and consecutive domains with opposite magnetization directions form a so-called strong-stripe phase. Compared to the Kittel model, in the present calculations (see reference 27 for more details), interactions between both surfaces of the film have been taken into account and a proper description of the magnetostatic energy of domains and Bloch-type domain walls is included. Within the domain walls, the angle of magnetization has been considered to vary linearly with position. Stripe domains have been assumed to be periodic in one direction and infinite and parallel in the other. This assumption is reasonable as the labyrinth structure changes to a parallel-stripe structure when a magnetic field is applied without stripe period modification. While this model is strictly speaking not valid at the onset of weak stripe, it becomes the best relevant for larger thicknesses. The total energy of the system results from a competition between magnetostatic, exchange and anisotropy energies. The total energy $\left(E_{S S}\right)$ in the strong stripe regime can be written under these approximations as:

$$
\begin{aligned}
E_{\mathrm{SS}}= & \frac{\mu_{0} d}{\pi t} \sum_{k=1}^{\infty}\left(\frac{\left|C_{k}\right|^{2}}{k} *\left(1-\mathrm{e}^{\frac{-\pi k t}{d}}\right)\right) \\
& +\frac{\pi^{2} A}{d \delta}+\frac{\delta K_{u}}{2 d} \\
C_{k}= & \frac{2 M_{S a t}}{k \pi\left(1-k^{2}\left(\frac{\delta}{d}\right)^{2}\right)} \cos \left[k \pi \frac{\delta}{2 d}\right]
\end{aligned}
$$

The analyzes of $M-H$ curves of the carbon doped $\mathrm{Mn}_{5} \mathrm{Ge}_{3}$ lead to a moderate value of $Q\left(Q_{\text {exp }}=0.2\right)$, which means that below a critical thickness, the magnetization is confined in the sample plane. Yet there is no direct transition between planar magnetization and strong-stripe domain structure. It rather occurs via an intermediate weak-stripe configuration. At the nucleation point (critical thickness for stripe nucleation), the magnetization within domains starts to be tilted out of the homogeneous in-plane direction leading to an alternating small deflection angle $(\theta)$ as schematically represented in Fig. 5. The canting angle $\theta$ varies from the maximum deflection angle $\left(\theta_{0}\right)$ to $-\theta_{0}$ over half a stripe period. The magnitude of $\theta_{0}$ continuously increases with the film thickness to finally reach $\pi / 2$, which defines the strong stripe regime. In order to describe this behavior, we have improved Saito's model ${ }^{22}$ by including magnetic interactions between top and bottom surfaces. The total energy $\left(E_{W S}\right)$ in the weak-stripe configuration is now given by:

$$
\begin{aligned}
E_{\mathrm{WS}}= & \frac{\mu_{0} d}{4 \pi t} \sum_{k=1}^{\infty}\left(\frac{\left|A_{k}\right|^{2}}{k} *\left(1-\mathrm{e}^{\frac{-\pi k t}{d}}\right)\right) \\
& +\frac{4 \theta_{0}^{2} A}{d^{2}}+\frac{K_{u}}{2}\left(1+\frac{\sin 2 \theta_{0}}{2 \theta_{0}}\right) \\
A_{k}= & \frac{-1^{k} 2 \theta_{0} M_{\text {Sat }} \cos \theta_{0}}{\frac{1}{4} k^{2} \pi^{2}-\theta_{0}^{2}}
\end{aligned}
$$

In equations (1) and (2) the saturation magnetization is denoted as $M_{\text {Sat }}$. $A$ and $K_{u}$ are the exchange stiffness and uniaxial anisotropy constants, respectively. $A_{k}$ and $C_{k}$ are Fourier coefficients describing the film magnetization, where $k$ is an odd number. For each experimental thickness $t$, numerical minimization with respect to two variables is performed. These are the period $d$ and the wall width $\delta$ in the strong stripe regime, and $d$ and $\theta_{0}$ in the weak stripe regime. Both energies, $E_{\mathrm{SS}}$ and $E_{\mathrm{WS}}$, are calculated and the lowest value is retained, which allows us to predict the nature of the magnetic configuration, namely either the weak- or the strong-stripe phase.

The saturation magnetization is set to the experimental value obtained at $300 \mathrm{~K}\left(640 \mathrm{kA} . \mathrm{m}^{-1}\right)$. On the basis of minimum energy arguments, we have calculated the stripe domain period considering the magnetocrystalline anisotropy and the exchange constants as adjustable parameters. The best fit to the experimental domain period is presented in the bottom panel of Fig. 5 and has lead to a magnetocrystalline anisotropy constant equal to 88 $\mathrm{kJ} / \mathrm{m}^{3}$ and an exchange stiffness $(A)$ equal to $2.4 \mathrm{pJ} / \mathrm{m}$. The micromagnetic coefficients $A$ and $K$ extracted from the modeling of stripes are of the same order of magnitude as those previously extracted, however close to $80 \%$ higher for $K$, and slightly less than double for $A$. Notice that the ratio $A / K$ is very similar in both cases. This is consistent with the fact that the critical thickness, related to $2 \pi \sqrt{(} A / K)$, is in the two approaches very similar $(30 \mathrm{~nm})$. While a naive model predicts the slope 2 for the period of stripes versus film thickness, ${ }^{26}$ our modeling should predict deviations from this law, both in slope and curvature. From these deviations may the absolute values of $A$ and $K$ be deduced. It is probable that the error bars in our measurements are still too large for a reliable analysis of these deviations. More precise measurements such as with magnetic scattering measurements may be required to go further. ${ }^{28}$ Our calculations predict also that a gradual transition from weak to strong magnetic configuration occurs between 28 and $44 \mathrm{~nm}$. Both features are in excellent agreement with the experimental results that show that a small out-of-plane component of the magnetization pointing alternatively upwards and downwards arises around $30 \mathrm{~nm}$. The predicted thickness corresponding to the change between weak- and strongstripe domain structure is $t=44 \mathrm{~nm}$. However, a complete study of the domain structure with thicker $\mathrm{Mn}_{5} \mathrm{Ge}_{3} \mathrm{C}_{0.7}$ films is still required to confirm this assumption. 


\section{CONCLUSION}

Using both magnetometric measurements and MFM domain imaging, we have identified the onset of a magnetization reorientation in thin epitaxial $\mathrm{Mn}_{5} \mathrm{Ge}_{3} \mathrm{C}_{0.7}$ films from in-plane to out-of-plane for a critical thickness of the order of $30 \mathrm{~nm}$. Beyond this critical thickness, a stripe domain structure is observed. This is in excellent agreement with the value calculated with our model that predicts a critical thickness of $28 \mathrm{~nm}$. Despite the discrepancy between the experimental and theoretical values of the magnetocrystalline anisotropy, its magnitude in both cases is considerably lower than the $(450 \pm 10)$ $\mathrm{kJ} / \mathrm{m}^{3}$ reported value for $\mathrm{Mn}_{5} \mathrm{Ge}_{3}$ thin films ${ }^{20}$ or for bulk $\mathrm{Mn}_{5} \mathrm{Ge}_{3} .{ }^{29}$ The seemingly minor introduction of $\mathrm{C}$ in the $\mathrm{Mn}_{5} \mathrm{Ge}_{3}$ lattice substantially impacts the magnetocrystalline anisotropy $\left(K_{u}\right)$. This drastic diminution may come from two sources. A first reduction of $K_{u}$ is probably introduced by the relative compressive stress according to $\Delta K_{u}=\frac{3}{2} \lambda \sigma$ where $\lambda$ and $\sigma$ are respectively the magnetostriction constant and the stress. Magnetoelastic constant has been measured in bulk monocrystalline samples to be $38 \times 10^{-6}$ at $15 \mathrm{~K} .^{30}$ Considering $0.9 \%$ stress and an elastic modulus of $110 \mathrm{GPa},{ }^{18}$ we estimate a decrease of about $60 \mathrm{~kJ} / \mathrm{m}^{3}$, which is not sufficient to explain the drastic change. The necessary stress yielding to the anisotropy variation would correspond to an unphysically $c$-axis compression of at least $7 \%$. Unlike in FePt-C where the reduction in $K_{u}$ is attributed to a clear deterioration in the chemical ordering, ${ }^{31}$ the crystallographic structure of $\mathrm{Mn}_{5} \mathrm{Ge}_{3}$ is barely affected by $\mathrm{C}$ incorporation.

The key to understand the change in magnetocrystalline anisotropy energy (MAE) probably lies in its origin that has been attributed by Van Vleck ${ }^{32}$ to the spinorbit coupling. In a first approximation, we consider the perturbation theory developed for the transition ferromagnetic metals; the uniaxial crystal anisotropy can be roughly estimated to be $\xi^{2} / W$, where $\xi$ is the spin orbit coupling (SOC) and $W$ is the $d$-band width near the Fermi energy. ${ }^{33}$ Band-structure calculations ${ }^{9}$ indicate a larger bandwidth especially in the case of $\mathrm{Mn}_{I I} 3 d$-band, which might partially explain the reduction of $K_{u}$. However, C-doping is likely to considerably lower the SOC due to hybridization effects between $\mathrm{Mn}$ and $\mathrm{C}$ atoms. Further investigation by ab initio calculations should be performed to confirm these assumptions.

In summary, we have investigated the magnetic properties of carbon-doped $\mathrm{Mn}_{5} \mathrm{Ge}_{3}$ thin films grown by MBE. Whereas the Curie temperature is greatly enhanced by the presence of $\mathrm{C}$, the latter reduces considerably the MAE. This feature is mainly assigned to hybridization effect between the Mn and $\mathrm{C}$ atoms. The critical thickness corresponding to the transition from in-plane to outof-plane magnetization is close to the one found in conventional uniaxial system such as Co for example. ${ }^{34}$ In other words, the singular character of $\mathrm{Mn}_{5} \mathrm{Ge}_{3}$ to form magnetic stripe domains for films as thin as $10 \mathrm{~nm}^{18}$ van- ishes through the introduction of $\mathrm{C}$ in the lattice in the favor of a higher Curie temperature.

\section{ACKNOWLEDGMENTS}

We would like to thank D. Dufeu (Inst. NEEL) for the technical support during the SQUID-VSM measurements. This work has been carried out thanks to the support of the A*MIDEX project (ANR-11-IDEX-000102) funded by the 'Investissements d'Avenir' French Government program, managed by the French National Research Agency (ANR).

${ }^{1}$ S. Picozzi, A. Continenza, and A. Freeman, Phys. Rev. B 70, 235205 (2004).

${ }^{2}$ A. Stroppa and M. Peressi, Materials Science in Semiconductor Processing 9, 841 (2006).

${ }^{3}$ R. P. Panguluri, C. Zeng, H. H. Weitering, J. M. Sullivan, S. C. Erwin, and B. Nadgorny, Physica Status Solidi (B) 242, R67 (2005).

${ }^{4}$ Y. S. Dedkov, M. Holder, G. Mayer, M. Fonin, and a. B. Preobrajenski, J. Appl. Phys. 105, 073909 (2009).

${ }^{5}$ W. Ndiaye, M. C. Richter, O. Heckmann, P. De Padova, J.-M. Mariot, a. Stroppa, S. Picozzi, W. Wang, a. Taleb-Ibrahimi, P. Le Fèvre, F. Bertran, C. Cacho, M. Leandersson, T. Balasubramanian, and K. Hricovini, Phys. Rev. B 87, 165137 (2013).

${ }^{6}$ A. Spiesser, H. Saito, R. Jansen, S. Yuasa, and K. Ando, Phys. Rev. B 90, 205213 (2014).

${ }^{7}$ I. A. Fischer, L.-T. Chang, C. Sürgers, E. Rolseth, S. Reiter, S. Stefanov, S. Chuissi, J. Tang, K. L. Wang, and J. Schulze, Appl. Phys. Lett. 105, 222408 (2014).

${ }^{8}$ M. Gajdzik, C. Su, M. T. Kelemen, and H. Lo, J. Magn. Magn. Mat. 221, 248 (2000).

${ }^{9}$ I. Slipukhina, E. Arras, P. Mavropoulos, and P. Pochet, Appl. Phys. Lett. 94, 192505 (2009).

${ }^{10}$ A. Spiesser, V. Le Thanh, S. Bertaina, and L. a. Michez, Appl. Phys. Lett. 99, 121904 (2011).

${ }^{11}$ C. Kittel, Phys. Rev. 70, 965 (1946).

${ }^{12}$ M. Hehn, S. Padovani, K. Ounadjela, and J. P. Bucher, Phys. Rev. B 54, 3428 (1996).

${ }^{13}$ M. Stoffel, L. Simona, J. L. Bischoff, D. Aubel, L. Kubler, and G. Casteleinb, Thin Solid Films 380, 32 (2000).

${ }^{14}$ A. Spiesser, I. Slipukhina, M.-T. Dau, E. Arras, V. Le Thanh, L. Michez, P. Pochet, H. Saito, S. Yuasa, M. Jamet, and J. Derrien, Phys. Rev. B 84, 165203 (2011).

${ }^{15}$ C. Sürgers, K. Potzger, and G. Fischer, J. Chem. Sci. 121, 173 (2009).

${ }^{16}$ M. Petit, L. Michez, C.-E. Dutoit, S. Bertaina, V. Dolocan, V. Heresanu, and . Le Thanh, Thin Solid Films 589, 427 (2015).

${ }^{17}$ A. Spiesser, M.-T. Dau, L. Michez, M. Petit, C. Coucreau, A. Glachant, and V. Le Thanh, International Journal of Nanotechnology 9, 428 (2012).

${ }^{18}$ A. Spiesser, F. Virot, L.-A. Michez, R. Hayn, S. Bertaina, L. Favre, M. Petit, and V. Le Thanh, Phys. Rev. B 86, 035211 (2012).

${ }^{19}$ H. M. Zhang, J. H. Grytzelius, and L. S. O. Johansson, Phys. Rev. B 88, 045311 (2013).

${ }^{20}$ L.-A. Michez, A. Spiesser, M. Petit, S. Bertaina, J.-F. Jacquot, D. Dufeu, C. Coudreau, M. Jamet, and V. Le Thanh, J. Phys.: Cond. Matt. 27, 266001 (2015).

${ }^{21}$ M. W. Muller, Phys. Rev. 122, 1485 (1961).

${ }^{22}$ N. Saito, H. Fujiwara, and Y. Sugita, J. Phys. Soc. Jpn 19, 1116 (1964).

${ }^{23}$ M. Seul and R. Wolfe, Phys.Rev. A 46, 7519 (1992).

${ }^{24}$ A. Kashuba and V. L. Pokrovsky, Phys. Rev. Lett. 70, 3155 (1993). 
${ }^{25}$ M. Hehn, R. Ferré, K. Ounadjela, and F. Rousseaux, J. Magn. Magn. Mat. 165, 5 (1997).

${ }^{26}$ A. Hubert and R. Schäfer, Magnetic Domains (Springer, BerlinHeidelberg-New York, 1998) p. 280.

${ }^{27}$ F. Virot, L. Favre, R. Hayn, and M. Kuz'min, J. Phys. D 45, 404003 (2012).

${ }^{28}$ H. A. Dürr, E. Dudzik, S. S. Dhesi, J. B. Goedkoop, G. van der Laan, M. Belakhovsky, C. Mocuta, and A. Marty, Science 284, 2166 (1999)

${ }^{29}$ G. Kappel, G. Fischer, and A. Jaegle, Phys. Lett. A 45, 267
(1973)

${ }^{30} \mathrm{~S}$. Barmin and A. A. Sevastyanov, FIZIKA TVERDOGO TELA 33, 3104 (1991).

${ }^{31}$ K. K. M. Pandey, N. Gaur, and C. S. Bhatia, J. Magn. Magn. Mat. 323, 2658 (2011).

${ }^{32}$ J. H. van Vleck, Phys. Rev. 52, 1178 (1937).

${ }^{33} \mathrm{P}$. Bruno, Physical Origins and Theoretical Models of Magnetic Anisotropy (IFF-Ferienkurs) (eds. P.H. Dederichs, P. Grünberg and W. Zinn,Jülich, 1993) pp. 1-28.

${ }^{34}$ J. Brandenburg, R. Hühne, L. Schultz, and V. Neu, Phys. Rev. B 79, 054429 (2009). 